\title{
Design and Verification of AC/DC Comparator Based on A/D Sampling
}

\author{
Yuanqiang Xiao \\ Kunming University of Science and Technology \& \\ Yunnan Power Grid Corp Graduate Workstations \\ Kunming, China \\ e-mail: 474324255@qq.com
}

\begin{abstract}
There is an important significance of AC/DC comparator with high precision for the precision measurement in electric power industry. An AC/DC comparator based on the non integer period Sampling and rapid data refactoring is designed. The AC/DC comparator consists of the input buffer, A/D change, DC reference standard, magnetic isolation and sampling control chip. AC/DC comparator is used to sample the AC Magnitude and realize the AC traceable to DC with the reference of the high precision DC reference standard. Through the error analysis of the AC/DC comparator for each part, the Maximum error of voltage amplitude measurement is $\pm 8 \times 10^{-6}$. The experimental results show that the accuracy of frequency, amplitude and phase is respectively $10^{-6} 、 10^{-6} 、 10^{-5}$, and the stability is $10^{-6} 、 10^{-6}$ 、 $10^{-5}$. It proves that the AC/DC comparator can accurate measurement the fundamental magnitude of $\mathrm{AC}$.
\end{abstract}

Keywords- AC/DC comparator; non integer period Sampling; data refactoring; $A / D$

\section{INTRODUCTION}

With the development of smart grid, the promoting of power grid information technology, the continuous application of high and new technology in electric power and the fine management of electric power, the electric power measurement technology and standards are increasingly more high requirements ${ }^{[1-3]}$. It is a practical significance to develop an AC/DC comparator which can compare AC voltage to $\mathrm{DC}$ voltage with high precision, because Voltage References are stored in the form of DC. The most commonly used AC/DC comparator are electric system $\mathrm{AC} / \mathrm{DC}$ comparator, electrostatic $\mathrm{AC} / \mathrm{DC}$ comparator and thermoelectric AC/DC comparator ${ }^{[4]}$. The relative error of electric system AC/DC comparator is level of $10^{-4}$ because of the residual charges, the inductance that exists between coils, swirl and electrostatic. The relative error of electrostatic AC/DC comparator is level of $10^{-5}$ because of the lifting wire impedance, the contact thermopower, the residual reactance of shunt, the surface charge and the different field strength. The thermoelectric AC/DC comparator is based on the principle of the same thermal effects between the AC voltage and DC voltage, but it must work with a high stability temperature environment, and the relative error of thermoelectric $\mathrm{AC} / \mathrm{DC}$ comparator is level of $2 \times 10^{-6}$.

A AC/DC comparator is designed, which use a high precision and high stability DC reference voltage as standard,

\author{
Min Cao, Bo Li, Dada Wang, Chuan Li, \\ Zhizhou Bi \\ Yunnan Electric Power Test \& Research Group CO, \\ LTD Electric Power Research Institute \\ Kunming, China \\ e-mail:cm1961@sohu.com
}

and the AC traceable $\mathrm{DC}$ is based on A/D's non integer period Sampling and rapid data refactoring. The AC/DC comparator's dependability is verified with experiment.

\section{NON-INTEGER-PERIOD SAMPLING AND DATA RECONSTRUCTION}

The sampling period Ts with A/D is given by

$$
T_{S}=\frac{K(1-\Delta) T_{0}}{N}
$$

Where $\Delta$ is the random number between -1 and $1, \mathrm{~T} 0$ is the period of the measured signal, $\mathrm{K}$ is the number of signal cycle in a sampling period and $\mathrm{N}$ is the number of sampling in a sampling period.

In the ideal situation, the periodic signal can be reconstructed at full period sampling to continuous periodic signal when $\Delta$ is $0, \mathrm{~K}$ and $\mathrm{N}$ are coprime number and $\mathrm{N}=\mathrm{L}+1^{[5-6]}$. Assumes that the sampling interval is $\Delta \mathrm{t}$ radians, and a cycle sampling points is $\Delta \mathrm{t}$ radians, and a cycle sampling points is $\mathrm{N}$, then $\Delta=\mathrm{tn}$. But $\Delta \mathrm{t}$ is not always zero. .Commonly used method is to adopt the method of phase lock loop dynamic tracking of measured signal to achieve the purpose of the whole cycle sampling ${ }^{[7]}$, but frequency tracking also appears a certain lag, cannot achieve high accuracy of measurement. Also some scholars put forward use the source table with bell technology, but the source being measured is not fixed, so it is hard to be totally with the lock. That is completely integral period sampling is done, but there's always some errors. Jiangqiu Zhang etc is theoretically proved that the innovation from the cycle model of a whole cycle sampling ${ }^{[8]}$.According to the principle, continuous signal is proposed using a whole cycle sampling strategy. Using liner interpolation method to a synchronous sampling sequence into a sequence of synchronous sampling, insert $2^{\wedge} \mathrm{N}$ points into a cycle. Using polynomial mathematical methods to piecewise polynomial fitting of the originnal sampling data, reconstruct fast Fourier transform of signal cycle integer point sequence.

\section{AC/DC COMPARATOR}

\section{A. The structure of AC/DC comparator}

An AC/DC comparator based A/D sampling and data processing technique is designed. $\mathrm{AC}$ instantaneous values are sampled by $\mathrm{A} / \mathrm{D}$, and the effective value, average value and etc can be acquire though calculating according to the 
definition of $\mathrm{AC}$ voltage. The structure of $\mathrm{AC} / \mathrm{DC}$ comparator is as shown in Figure 1.

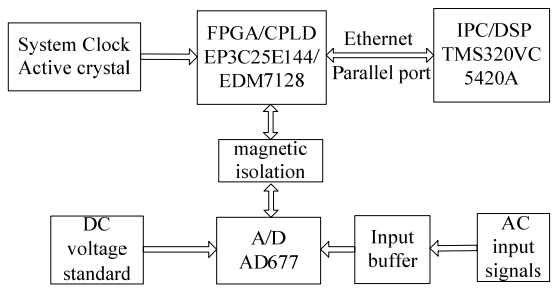

Figure 1. The structure of AC/DC comparator

The AC/DC comparator is composed of input buffer, $\mathrm{A} / \mathrm{D}$ conversion, the DC voltage standard, magnetic isolators, the processor and the system clocks, and it can link to IPC by Ethernet and parallel port. The AC input signals compare with DC voltage reference through $\mathrm{A} / \mathrm{D}$ after input buffer and the signal is collected and processed by data capture system, and $\mathrm{AC}$ voltage trace to the $\mathrm{DC}$ voltage though in this way. The A/D AD677 is 16 bit which has selfcalibration function. Its sample frequency is $50 \mathrm{~Hz}$. The valid value of AC voltage $U_{A C}$ is given by:

$$
Y_{A X}=\kappa \cdot Y_{\Delta X}
$$

Where the $U_{D C}$ is DC reference voltage, $k$ is the conversion factor of $\mathrm{AC} / \mathrm{DC}$.

\section{B. Experimental verification system}

Experimental verification system is composed of DC reference voltage reference, $\mathrm{AC}$ voltage source, Precision resistors, current transformer, $\mathrm{AC} / \mathrm{DC}$ comparator and DSP (digital signal processor). As shown in Figure 2.

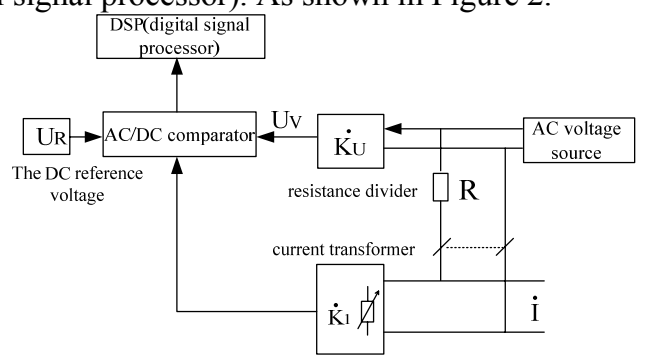

Figure 2. The composition of experimental verification system

And the DC reference voltage reference, AC voltage source, precision resistors are standard component. The precision resistor is designed with No inductance and capacitance. The AC/DC comparator is zero calibration and full-scale calibration. DC reference standard of $\mathrm{AC} / \mathrm{DC}$ comparator compare to $\mathrm{DC}$ reference voltage reference by eight semi-digital digital multimeters. Temperature Coefficient of precision resistors is $0.1 \mathrm{ppm} /{ }^{\circ} \mathrm{C}$.

\section{ERROR ANALYSIS}

The main sources of error is voltage measurement error. And it is composed of the error by DC reference voltage, sampling method, input buffer and A/D converter.

\section{A. The error by DC reference voltage}

The error by DC reference voltage is caused by measurement error which is internal of $\mathrm{AC} / \mathrm{DC}$ comparator. The DC reference voltage is saved by Fluke732B with standard group. The uncertainty of DC reference voltage measurement is $5.0 \times 10^{-7}$, and the measurement error $E_{r r 1}$ of $7 \mathrm{~V}$ DC voltage is $1.75 \times 10^{-6} \mathrm{~V}$.

The DC reference which is internal of $\mathrm{AC} / \mathrm{DC}$ comparator is LTZ1000ACH, and its annual variation of technical index is $0.83 \times 10^{-6}$. So the maximum error $E_{r r 2}$ which is caused by DC reference changes is $5.81 \times 10^{-6} \mathrm{~V}$. When $E_{r r 1}$ and $E_{r r 2}$ are limit value, though line synthesis, the quantity transfer error of DC reference voltage Err is $7.56 \times 10^{-6} \mathrm{~V}$ calculated by $E_{r r 1}+E_{r r 2}$. And the relative error $E$ is $1.1 \times 10^{-6}$.

According to the principle of $\mathrm{A} / \mathrm{D}$ converter, $\mathrm{AC} 4 \mathrm{~V}$ measurement error $\Delta U_{l}$ which compare to DC reference voltage is calculated by:

$$
\Delta U_{I}=4 \mathrm{~V} \times E=4 \mathrm{~V} \times 1.08 \times 10^{-6}=4.32 \times 10^{-6} \mathrm{~V}
$$

\section{B. The error of Sampling algorithm}

According to the principle of non integer period Sampling ${ }^{[9]}$, In a period of $\mathrm{N}=1000$ points, the relative error $\delta_{r m s l}$ of sampling algorithm is $6.6 \times 10^{-6}$.

According to the principle of backlash compensation algorithm, in the condition of continuously sampling ten period, the relative error $\delta_{r m s 2}$ is $0.07 \times 10^{-6}$.

In conclusion, at the full-scale input $4 \mathrm{~V}$, the error of arithmetic $\Delta U_{2}$ is calculated by:

$$
\Delta U_{2}=4 \mathrm{~V} \times 0.1 \times 10^{-6}=0.4 \times 10^{-6} \mathrm{~V}
$$

\section{The error of input buffer}

The AC input buffer of AC/DC comparator is an inphase follower which is composed of High Precision Operational Amplifiers OP27E, and the error of input buffer $E_{b u f}$ is given by:

$$
E_{\beta \nu \phi}=-1 /(\mathrm{K}+1)
$$

Where $\mathrm{K}$ is Open-loop gain of Operational Amplifier.

The $\mathrm{K}$ is $1.8 \times 10^{6}$, according to manufacturer of OP27E., the error of input buffer $E_{b u f}$ is $-0.56 \times 10^{-6}$ by form (3).

But the Open-loop gain $K$ is a changed value, the changing ranges is set to $10 \%$. According to the form(3), the Open-loop gain of Operational Amplifier $K$ is $1.8 \times 10^{6}, K_{l}$ which is after changing $10 \%$ is $1.62 \times 10^{6}$. The modulus of changing of input buffer error $\Delta E_{\text {buf }}$ is $0.06 \times 10^{-6}$ calculated by $\left|\left[-1 /\left(K_{1}+1\right)\right]-[-1 /(K+1)]\right|$.

From Above Analysis and considering converted quantity of Operational Amplifiers, When $\Delta E_{b u f}$ are limit value, the error of input buffer $E$ is $-0.62 \times 10^{-6}$.

In conclusion, at the full-scale input $4 \mathrm{~V}$, the error of input buffer $\Delta U_{3}$ is calculated by:

$$
\Delta U_{3}=4 \mathrm{~V} \times\left(-0.62 \times 10^{-6}\right)=-2.48 \times 10^{-6} \mathrm{~V}
$$

\section{$D$. The error of $A / D$ converter}

The $\mathrm{A} / \mathrm{D}$ of $\mathrm{AC} / \mathrm{DC}$ comparator is 16 bit, the resolution error of AC sampling $E_{n}$ is $0.12 \times 10^{-6}$. The DC calibration 
target of $\mathrm{A} / \mathrm{D}$ standard deviation is a fixed value, and it is $1 / 4 \mathrm{LSB}$. Reducing the converter error to $1 / 12 \mathrm{LSB}\left(1.25 \times 10^{-}\right.$ $\left.{ }^{6}\right)$. the error of the DC voltage calibration $E_{d}$ is $1.25 \times 10^{-6}$.

When $E_{n}$ and $E_{d}$ are limit value, though line synthesis, the resolution error of AC sampling $E_{n d}$ is $1.37 \times 10^{-6}$ calculated by $E_{n}+E_{d}$. The system error of AC sampling $E_{s}$ is estimated at half of the energy levels which is $3 \times 10^{-6}$ after it is corrected.

The error of $\mathrm{A} / \mathrm{D}$ convert $E_{A D}$ consists of the resolution error of $\mathrm{AC}$ sampling $E_{n d}$ and the system error of $\mathrm{AC}$ sampling $E_{s}$, and the $E_{n d}$ and $E_{s}$ is independent. When $E_{n d}$ and $E_{s}$ are limit value, though line synthesis, the error of $\mathrm{A} / \mathrm{D}$ convert $E_{A D}$ is $4.37 \times 10^{-6}$ calculated by $E_{n d}+E_{s}$.

In conclusion, at the full-scale input $4 \mathrm{~V}$, the error of $\mathrm{A} / \mathrm{D}$ convert $\Delta U_{4}$ is calculated by:

$$
\Delta U_{4}=4 \mathrm{~V} \times\left(4.37 \times 10^{-6}\right)=1.748 \times 10^{-5} \mathrm{~V}
$$

\section{E. The total error}

From above error analysis, when the error of input buffer is not corrected by the algorithmic, the AC measuring error of $\mathrm{AC} / \mathrm{DC}$ comparator $\Delta U$ is given by:

$$
\otimes Y=\otimes Y_{1}+\otimes Y_{2}+\otimes Y_{3}+\otimes Y_{4}
$$

According to equation (4), the $\Delta U$ is $24.32 \times 10^{-6} \mathrm{~V}$.In conclusion, at the full-scale input $4 \mathrm{~V}$, the relative error of AC voltage measuring $\Delta$ is $6.08 \times 10^{-6}$.

Thinking about the effect of the board layouts and the testing circuit, when all values is limited, the error estimate of AC/DC comparator voltage measuring is not over $2 \times 10^{-6}$. So the maximum error of $\mathrm{AC}$ voltage measuring with AC/DC comparator is $\pm 8 \times 10^{-6}$.

\section{TESTING AND ANALYSIS}

The stability and accuracy of AC voltage, phase and frequency is tested in a constant temperature and humidity environment(the temperature is $20^{\circ} \mathrm{C}$, the relative humidity is $60 \% \mathrm{rh}$ ). Standard AC $4 \mathrm{~V}$ signal of $50 \mathrm{~Hz}, 52.45 \mathrm{~Hz}, 54.94 \mathrm{~Hz}$, $57.4 \mathrm{~Hz}, 59.88 \mathrm{~Hz}, 62.34 \mathrm{~Hz}$ and $64.8 \mathrm{~Hz}$ is respectively input to $\mathrm{AC} / \mathrm{DC}$ comparator, and the phase of input signal is respectively $0^{\circ} 、 60^{\circ} 、 180^{\circ}$ and $300^{\circ}$. The AC voltage is measured by AC/DC comparator, as shown in Figure 3.

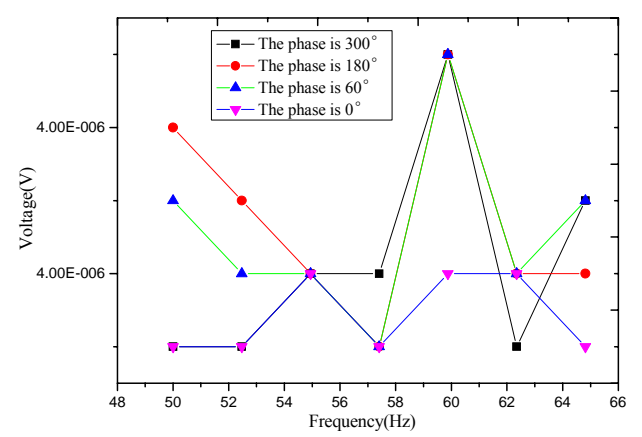

Figure 3. Measuring $4 \mathrm{~V}$ under different phase and frequency

In figure 3 , the maximum difference of voltage measurement is $4 \times 10^{-7} \mathrm{~V}$ when the input voltage is at different phase, and the maximum difference of voltage measurement is $3 \times 10^{-7} \mathrm{~V}$ when the input voltage is at same frequency and different phase.

\section{A. Stability test}

The frequency stability of input voltage is tested by $\mathrm{AC} / \mathrm{DC}$ comparator. The data is recorded once every hour, and every time record five data. The mean value of five data is as the measuring value, and there are seven sets of data. The frequency stability curve is draw through calculation, as shown in Figure 4.

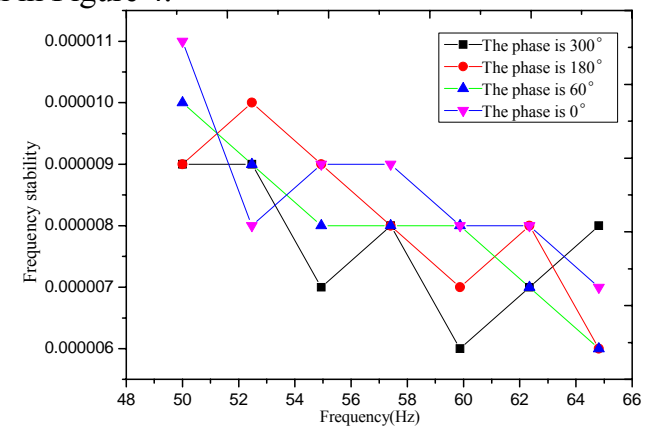

Figure 4. Frequency stability curve under different frequency and different phase

In figure 4, Frequency stability of AC/DC comparator is gradually drop off with increasing frequency. Frequency stability of AC/DC comparator is the level of $10^{-6}$ as a whole.

Voltage magnitude stability is tested in the same way, and the voltage magnitude stability curve is draw, as shown in figure 5.

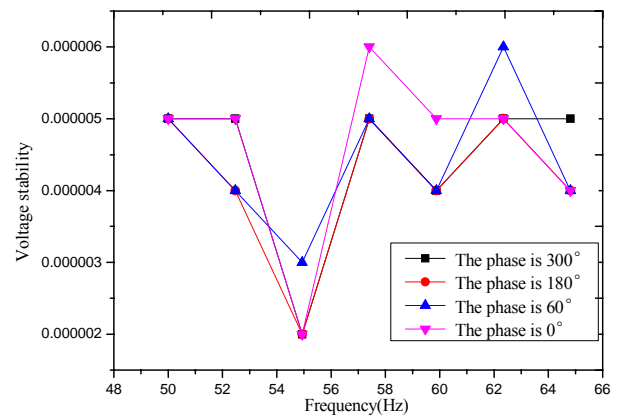

Figure 5. Voltage magnitude stability curve under different frequency and different phase

In figure 5, the voltage magnitude stability has an obviously minimal value in $55 \mathrm{~Hz}$ at every phase. Voltage magnitude stability value of $\mathrm{AC} / \mathrm{DC}$ comparator is the level of $10^{-6}$ as a whole. The maximum value is $6 \times 10^{-6}$, and the minimum value is $2 \times 10^{-6}$.

Phase stability of AC/DC comparator is tested in the same way, and the phase stability curve is draw, as shown in figure 6 . 


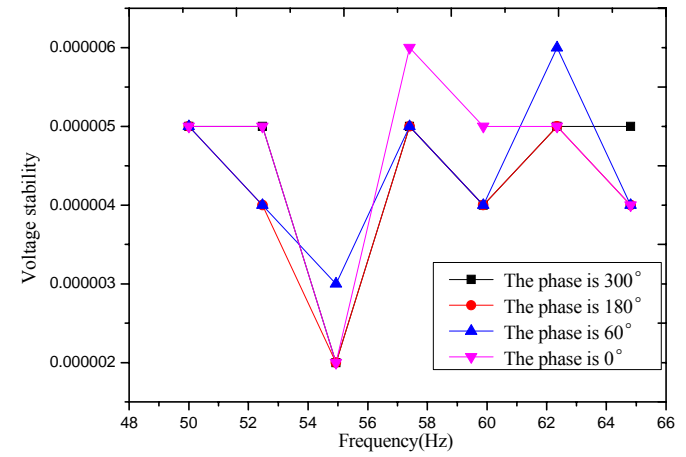

Figure 6. Phase stability curve under different frequency and different phase

In figure 6 , the phase stability has an obviously minimal value in $55 \mathrm{~Hz}$ at every phase, and the maximum value is $5 \times$ $10^{-5}$, and the minimum value is $2 \times 10^{-5 \circ}$.

\section{B. Accuracy test}

The accuracy of AC/DC comparator is test with different phase and different frequency voltage. Standard AC 4V signal of different phase and different frequency is generate by a standard source. The data tested by AC/DC comparator is recorded once every hour, and every time record five data. The mean value of five data is as the measuring value at that point, and there are seven sets of data. The frequency accuracy curve is draw through calculation, as shown in Figure 7

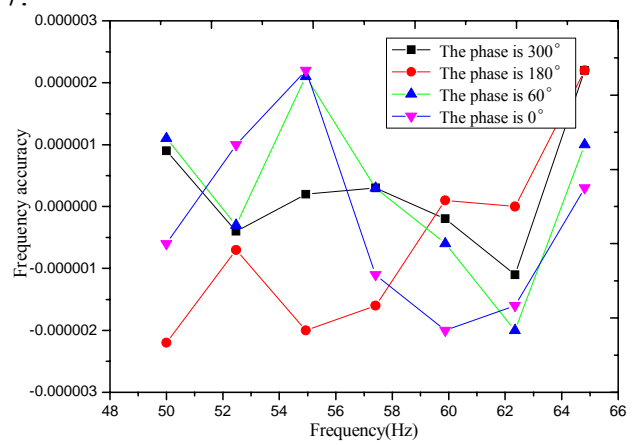

Figure 7. Frequency accuracy curve under different frequency and different phase

In figure 7 , the different value is minimum at $65 \mathrm{~Hz}$, frequency accuracy of AC/DC comparator is the level of $10^{-6}$ as a whole, and the maximum value is $2.3 \times 10^{-6}$, and the minimum value is $-2.2 \times 10^{-6}$.

Voltage magnitude accuracy of AC/DC comparator is tested in the same way, and the voltage magnitude accuracy curve is draw, as shown in figure 8 .

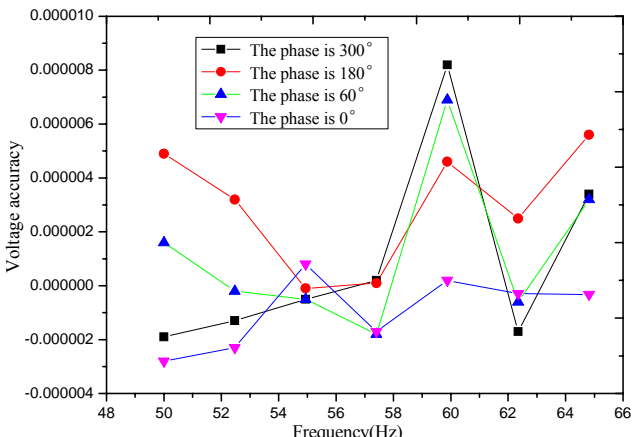

Figure 8. Voltage magnitude accuracy curve under different frequency and different phase

In figure 8 , and the maximum value of voltage magnitude accuracy is $2.3 \times 10^{-6}$, and the minimum value of voltage magnitude accuracy is $-2.2 \times 10^{-6}$.

Phase accuracy of AC/DC comparator is tested in the same way, and the phase accuracy curve is draw, as shown in figure 9.

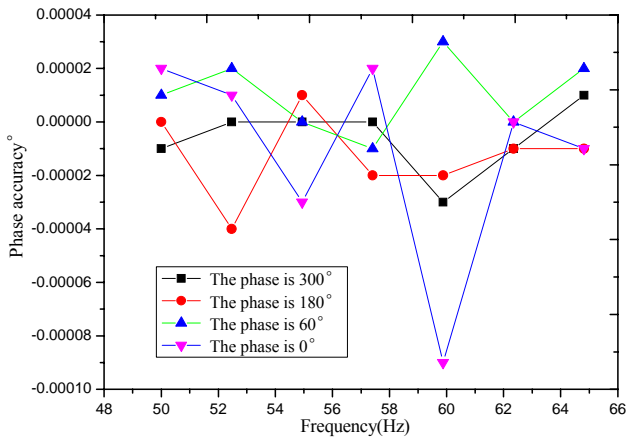

Figure 9. Phase accuracy curve under different frequency and different phase

In Figure 9, the different value is maximum which is 12 $\times 10^{-5 \circ}$ at $60 \mathrm{~Hz}$, and the maximum value of phase accuracy is $3 \times 10^{-5 \circ}$, and the minimum value of phase accuracy is -9 $\times 10^{-5 \circ}$.

\section{SUMMARY}

Structure of the AC/DC comparator are introduced, and the voltage error of $\mathrm{AC} / \mathrm{DC}$ comparator is $\pm 8 \times 10^{-6}$ in theory. The stability and accuracy of phase, frequency and voltage magnitude is tested. Tests show that the stability of phase, frequency and voltage magnitude are respectively the level of $10^{-6}, 10^{-6}, 10^{-5}$, and the accuracy of phase, frequency and voltage magnitude are respectively the level of $10^{-6}, 10^{-6}, 10^{-}$ ${ }^{5}$.It proves that the AC/DC comparator can reach highprecision measurement of basic electrical parameters.

\section{REFERENCES}

[1] S J. XIAO, "Consideration of Technology for Constructing Chinese Smart Grid," Automation of electric power systems, vol. 33, May. 2009, pp. 1-4.

[2] L. Jingjun and D. Zemei and Z. Li, "On-line power system disturbance monitoring and evaluation method based on WAMS," Automation of electric power systems, vol.36, Apr. 2012, pp.82-86 
[3] D. Weiwu, and P. Tianhong, and L. Zhengming, "Design of Power Synthesized Parameters Monitor for Power Demand Side, ', Instrument technique and sensor, Jul.2012, pp. 26-28.

[4] Z. Xinming, and Z. Yin, "Study on very-low frequency AC/DC comparator," Chinese journal of scientific instrument, Vol.13, May. 1992, pp. 125-129.

[5] Z. Jianqiu, and T. Ran, "The application of non-integer-period sampling method in spectrum analysis of periodic signals," Chinese journal of scientific instrument, Vol.16, Feb.1995, pp. 1-7.

[6] X. Deyin, X. Pingfang, and F. Zhihong, "Error analysis of noninteger-period sampling for electric sinusoidal signal," Journal of electronic measurement and instrument, vol.24, Feb.2010, pp.113-118.
[7] Y. Zhicang, S. Qiang, and L. Wenhua, "A modified soft phase lock loop algorithm improving the performance in dynamic phase tracking and detectiom of unbalanced voltage," Power system technology, vol. 34, Jan.2010, pp. 31-35.

[8] Z. Jianqiu, and S. Yi, "The non-integer-period sampling theory for periodic siqnals," Journal of HARBIN institute of technology, Vol. 27, Dec.1995, pp.99-103.

[9] G. Liang, Z. Dongshen, "Backlash compensation on CNC machine tool based on semi-closed loop control," Apr. 2011, pp.47-50. 